\title{
SIMULATION OF CONVECTION AND RIPENING IN A BINARY ALLOY MUSH USING THE PHASE-FIELD METHOD
}

\author{
H.-J. DIEPERS ${ }^{1}$, C. BECKERMANN ${ }^{1} \uparrow$ and I. STEINBACH ${ }^{2}$ \\ 'Department of Mechanical Engineering, University of Iowa, Iowa City, IA 52242, U.S.A. and \\ ${ }^{2}$ ACCESS e.V., Intzestraße 5, 52056 Aachen, Germany
}

(Received 7 June 1999; accepted 25 June 1999)

\begin{abstract}
Two-dimensional Ostwald ripening of an $\mathrm{Al}-4 \% \mathrm{Cu}$ alloy solid/liquid mush in the presence of melt convection, and the influence of ripening on the flow, is studied numerically using a recent extension of the phase-field method that accounts for flow in the liquid phase. Through a parametric study, the ripening kinetics are investigated and compared for cases with and without melt convection. The cases without convection show good agreement with available coarsening theories for a finite fraction of solid. In the cases with flow the mean radius of the solid particles increases at a faster rate than without convection. The ripening exponent changes from $1 / 3$ to $1 / 2$, while the rate constant depends on the fraction of solid. Comparisons are made with the convective ripening theory of Ratke and Thieringer. Although the present analysis of coarsening is hampered by the limited number of particles in the domain, some qualitative results are presented for the effect of convection on the particle radius distribution. Finally, the present simulations allow for a determination of the permeability of the mush as a function of the fraction of solid, and the dependence of the permeability on the ripening kinetics is shown to be scalable using the specific surface area or the mean radius. (C) 1999 Acta Metallurgica Inc. Published by Elsevier Science Ltd. All rights reserved.
\end{abstract}

Keywords: Ripening; Casting; Alloys, aluminum; Microstructure; Theory and modeling, kinetics, transport, diffusion

\section{INTRODUCTION}

One of the most challenging problems in modeling of solidification of metal alloys is the mutual interaction between physical phenomena occurring on different length scales. In particular, the macroscopic transport processes in a solid/liquid mushy zone are closely coupled to the evolution of the solid morphology on a microscopic scale. Several methods allow for the incorporation of a variety of microscale phenomena in the macroscopic transport equations [1-4]. In any of these methods, a key factor in determining the extent of macroscale solute and heat transport is the modeling of convection in the mushy zone. Interdendritic melt flow is like a flow through a porous medium, and the permeability $\Pi$ of the mush, as defined in Darcy's law

$$
U=-\Pi \frac{\nabla p}{\eta}
$$

is often used to correlate the mean or superficial velocity $U$ through a mush volume with the pressure gradient $\nabla p$ and the dynamic viscosity $\eta$. The permeability generally depends on the size and geometry of the microscopic flow channels formed by

$\dagger$ To whom all correspondence should be addressed. the growing solid, and may be anisotropic [5-9]. The channel geometry is often characterized by the specific surface area $S_{\mathrm{V}}$ (=solid/liquid interfacial area per volume of solid) or the hydraulic radius $R_{\mathrm{h}}$. For a given fraction of liquid $f_{1}$ in a representative mush volume, the permeability can vary strongly due to changes in the morphology of the microstructure. Once the initial microstructure is established, Ostwald ripening [10] is the main mechanism by which such changes in the morphology can occur. Ripening (or coarsening) generally causes the specific surface area to decrease with time, and hence the permeability to increase. It is important to realize that Ostwald ripening can be influenced by convection in the mush. In other words, the morphology of the mush not only governs the flow, but the flow also influences the evolution of the morphology. This interplay between flow and Ostwald ripening in a mush is the subject of the present study.

Models for the permeability of a mush are often based on the Blake-Kozeny equation, where the hydraulic radius is correlated to the primary or secondary dendrite arm spacings. Theoretical studies have relied on analytical solutions describing the flow of a viscous fluid through regular arrays of cylinders to deduce the permeability $[11,12]$. 
Numerical simulations of flow through arrays of cruciforms have also been used to establish models for the permeability of a mush [13]. These approaches still need calibration through experimental data, which are not available for small and high liquid volume fractions $[8,9]$. In these regimes experimental methods fail due to the fragility of the dendrites or ripening effects. In order to escape this dilemma, Bhat et al. [8] have simulated directly the flow through a microstructure in two dimensions, where the solid geometry was taken from digitized images of a real mush. All previous studies take the solid morphology to be fixed and do not consider interactions between the flow and the evolving microstructure.

The ripening effect was first described in 1900 by Ostwald [10]. It is the main mechanism by which the higher order dendrite arms adjust their spacing in a solidifying mush. Ripening occurs near thermodynamic equilibrium through a reduction in the surface free energy. The different curvature undercoolings of the various sized particles in a mush lead to concentration gradients (in an alloy) in the melt, causing the particles to exchange solute such that the larger particles grow at the expense of the smaller ones. The first quantitative analysis of ripening in the limit of purely diffusive transport and a vanishing volume fraction of solid was provided by Lifshitz, Slyozov and Wagner (LSW) [14, 15]. This theory shows that the particle radii achieve a stationary, self-similar distribution and that the mean radius, $R$, grows with time, $t$, according to

$$
R^{3}-R_{0}^{3}=K_{\text {diff }} t
$$

or in the long-time limit

$$
R=\left(K_{\text {diff }} t\right)^{1 / 3}
$$

where $R_{0}$ is the initial mean radius and $K_{\text {diff }}$ is the diffusion ripening rate constant. Similarly, the specific surface area can be shown to decrease with the cube root of time [16]. Several extensions of this theory have been proposed to take into account the effect of a finite volume fraction of the dispersed phase, different dimensionalities of the ripening system, and coupled thermal and solutal transport [17, 18]. In general, these extensions again lead to a selfsimilar radius distribution and a ripening exponent of $1 / 3$, but there are differences in the radius distribution functions and the rate constants (for twodimensional results see Refs [19-21]). Experiments have confirmed the general ripening laws in terms of self-similarity and the ripening exponent. However, present theories are unable to account for the transient behavior of a ripening system or coalescence of particles which can occur at higher volume fractions of the dispersed phase [17].

Several studies have been performed to investigate the effect of relative movement between the solid particles and the melt on ripening. A theoretical analysis of ripening in the presence of moving particles was first presented by Ratke and Thieringer [22]. Particle motion leads not only to changes in the radius distribution, but also in the ripening exponent (it changes from $1 / 3$ to $1 / 2$ ). This tendency has been verified in experiments [23]. Ripening dominated by convection has also been studied by Akaiwa et al. [24] and Wan and Sahm [25].

Recently, a number of numerical simulation methods have been developed to investigate ripening problems [21, 26, 27]. For example, Küpper and Masbaum [27] solve numerically the Cahn-Hilliard equation. While such simulations show much promise in analyzing more complex ripening systems, they often employ simplifying assumptions. To our best knowledge, all previous numerical simulations of ripening are limited to purely diffusive transport of heat or species.

The present study of coupled ripening and melt flow in a binary alloy mush is based on numerical simulations employing the phase-field method. This model has a broad theoretical basis and can be derived starting with a free energy functional of the Ginzburg-Landau type that includes not only phase related free energies, but also interfacial contributions. Langer [28] first applied the phase-field method to solidification problems. Kobayashi contributed much to the popularity of the phase-field method by simulating the growth of thermal dendrites [29]. Several studies have since improved the theoretical foundation [30] and accuracy [31] of the method. Recent applications include solidification of alloys [32, 33] and other multiphase, multicomponent systems [34]. In the phase-field method, an order parameter (or phase indicator function) $\phi$ is introduced that varies smoothly from one constant value in the solid phase (i.e. 0) to another constant value in the liquid phase (i.e. 1) across a thin, but finite and numerically resolvable layer representing the solid-liquid interface. The phase-field equation is used to propagate $\phi$ in space and time. The phase-field method completely avoids the explicit calculation of interface normals and curvatures, which are needed in alternative methods that are based on front-tracking together with explicit satisfaction of the Gibbs-Thomson condition at the solid-liquid interface. The present authors have recently extended the phase-field method to include convection in the liquid phase $[35,36]$. The only application of the phase-field method to ripening reported in the literature is the study of Warren and Murray [37] for a binary alloy in two dimensions. While their study demonstrates the potential of the phase-field method in simulating Ostwald ripening, it is limited to purely diffusive solute transport and no results are reported for the longtime, stationary ripening behavior, preventing a detailed comparison with available theories. 
In the following we provide a brief overview of the governing equations and numerical solution procedures employed in the present study. Then, detailed numerical results are presented for twodimensional ripening of a solid-liquid mixture (or mush) of a solidifying $\mathrm{Al}-\mathrm{Cu}$ alloy both under diffusive and convective conditions. The ripening results without and with convection are compared with existing mean-field theories. Finally, we present results for the effect of ripening on the permeability of the mush, and compare them with other available data.

\section{GOVERNING TRANSPORT EQUATIONS}

A detailed derivation of the present phase-field model with melt convection is published elsewhere $[35,36]$, and only a brief summary is provided here. The governing transport equations consist of the evolution equation for the phase field, the species conservation equation, and the mass and momentum conservation equations. These equations are part of a more general phase-field model that can be used to predict a variety of solidification microstructures [36]. The energy equation is specific to the system considered here and is presented in the next section.

The phase distribution is described by the order parameter $\phi$, which changes smoothly from $\phi=0$ in the liquid to $\phi=1$ in the solid across a diffuse interface layer of thickness $6 \delta$. We assume a stationary front profile of the form $\phi(x)=$ $1 / 2[1-\tan h(x / 2 \delta)]$ across the diffuse interface. This profile is transported by the Gibbs-Thomson condition at the interface $T=T_{\mathrm{m}}+m_{1} C_{1}-\Gamma \kappa-v / \mu$, where $T$ is the temperature, $T_{\mathrm{m}}$ is the melting point of the pure substance, $C_{1}$ is the concentration in the liquid, $m_{1}$ is the liquidus slope from the equilibrium phase diagram, $\kappa$ is the curvature of the liquid/solid interface, $\Gamma=\sigma / \Delta S_{\mathrm{f}}$ is the Gibbs-Thomson coefficient given by the ratio of the surface tension $\sigma$ to the entropy of fusion $\Delta S_{\mathrm{f}}, v$ is the normal interface velocity, and $\mu$ is the linear kinetic coefficient. 36]

The resulting phase-field equation is given by [35,

$$
\begin{aligned}
& \frac{\partial \phi}{\partial t}=\mu \Gamma\left(\nabla^{2} \phi-\frac{\phi(1-\phi)(1-2 \phi)}{\delta^{2}}\right) \\
&+\frac{\mu}{\delta} \phi(1-\phi)\left(T-T_{\mathrm{m}}-m_{1} C_{1}\right) .
\end{aligned}
$$

The species conservation equation is written in terms of a mixture concentration $C$ defined as $C=(1-\phi) C_{1}+\phi C_{\mathrm{s}}$, where $C_{\mathrm{s}}$ is the concentration in the solid. Assuming equilibrium partitioning inside the diffuse interface layer, i.e. $C_{\mathrm{s}}=k C_{1}$ (where $k$ is the partition coefficient), the solute balance is given by $[35,36]$

$$
\begin{aligned}
& \frac{\partial C}{\partial t}+\nabla \cdot\left(\frac{1-\phi}{1-\phi+k \phi} \mathbf{v}_{1} C\right) \\
& =\nabla \cdot \tilde{D}\left[\nabla C+\frac{(1-k) C}{1-\phi+k \phi} \nabla \phi\right] \\
& \text { with } \tilde{D}=\frac{(1-\phi) D_{1}+k \phi D_{\mathrm{s}}}{1-\phi+k \phi}
\end{aligned}
$$

where $D_{\mathrm{s}}$ and $D_{1}$ are the mass diffusivities in the solid and liquid, respectively, and $\mathbf{v}_{1}$ is the intrinsic velocity of the liquid. The second term on the lefthand side of equation (5) accounts for the advective flux of species in the liquid, while the right-hand side includes the species fluxes due to diffusion and interfacial segregation. The above species conservation equation is valid in the entire domain regardless of the phase present, and it can easily be seen that it reduces to the correct forms in the singlephase liquid $(\phi=0)$ and solid $(\phi=1)$ regions.

The mass and momentum conservation equations, assuming equal densities, $\rho$, of the solid and liquid phases and a stationary rigid solid, are given by $[35,36]$

$$
\begin{gathered}
\nabla \cdot(1-\phi) \mathbf{v}_{1}=0 \\
\frac{\partial}{\partial t}\left[(1-\phi) \mathbf{v}_{1}\right]+\nabla \cdot\left[\mathbf{v}_{1}(1-\phi) \mathbf{v}_{1}\right] \\
=-\frac{1}{\rho}(1-\phi) \nabla p_{1}+v \cdot \nabla^{2}\left[(1-\phi) \mathbf{v}_{1}\right] \\
-h \frac{v \phi^{2}(1-\phi)}{\delta^{2}} \mathbf{v}_{1}
\end{gathered}
$$

where $p_{1}$ is the pressure and $v$ is the kinematic viscosity of the liquid. The above equations are valid in the single-phase liquid and the diffuse interface region; in the solid, the solution of the momentum equation is $\mathbf{v}_{1}=0$. The last term in equation (7) is an interfacial stress, which gradually forces the velocity to zero as the fully solid region is approached. The constant $h$ is chosen such that for plane flow past the interface the velocity profile outside the smeared interface matches the one for a sharp interface with a no-slip condition at $\phi=0.5$. A value of $h=2.757$ was determined from an asymptotic analysis [36], and was shown to be independent of the diffuse interface thickness.

\section{SIMULATION CONDITIONS AND SOLUTION PROCEDURES}

We consider ripening of a solid/liquid mixture of an $\mathrm{Al}-\mathrm{Cu}$ alloy of concentration $C_{0}=4 \mathrm{wt} \%$ inside a two-dimensional, square domain of size $\mathrm{L} \times \mathrm{L}=0.9 \times 0.9 \mathrm{~mm}^{2}$. The domain can be thought of as a representative cross sectional area element of a mushy zone. Initially, spherical solid particles exist randomly in the liquid. The initial volume fraction of solid in the domain is varied. The particles are fixed in space and their initial size distri- 
Table 1. Thermophysical properties of $\mathrm{Al}-4 \mathrm{wt} \% \mathrm{Cu}$ alloy used in the simulations [38, 39]; for practical reasons the value of $\delta$ was chosen to be approximately equal to the length of one grid cellthe actual interface thickness, $6 \delta^{\text {act. }}$, is of the order of $10^{-9} \mathrm{~m}$; the actual kinetic coefficient, $\mu_{\mathrm{k}}^{\text {act. }}$, is approximately $0.33 \mathrm{~m} / \mathrm{s} \mathrm{K}$-the value in the table was obtained by multiplying $\mu_{\mathrm{k}}^{\text {act. }}$ by the ratio $\delta^{\text {act. }} / \delta$

\begin{tabular}{lc}
\hline Property & Value \\
\hline$T_{\mathrm{m}}$ & $933.6 \mathrm{~K}$ \\
$m_{1}$ & $2.6 \mathrm{~K} / \%$ \\
$\Gamma$ & $2.41 \times 10^{-7} \mathrm{mK}$ \\
$D_{1}$ & $3 \times 10^{-9} \mathrm{~m}^{2} / \mathrm{s}$ \\
$D_{\mathrm{s}}$ & $3 \times 10^{-13} \mathrm{~m}^{2} / \mathrm{s}$ \\
$k$ & 0.14 \\
$L$ & $9.5 \times 10^{8} \mathrm{~J} / \mathrm{m}^{3}$ \\
$\rho c_{p}$ & $2.58 \times 10^{6} \mathrm{~J} / \mathrm{K} \mathrm{m}$ \\
$\rho$ & $2.475 \times 10^{3} \mathrm{~kg} / \mathrm{m}^{3}$ \\
$\eta$ & $0.014 \mathrm{poise}$ \\
$6 \delta$ & $1.27 \times 10^{-5} \mathrm{~m}$ \\
$\mu$ & $2.6 \times 10^{-5} \mathrm{~m} / \mathrm{s} \mathrm{K}$ \\
\hline
\end{tabular}

bution is set according to the two-dimensional coarsening theory of Marqusee [20]. The thermophysical properties of the $\mathrm{Al}-4 \mathrm{wt} \% \mathrm{Cu}$ alloy are summarized in Table 1. Note that the mass diffusivities $D_{1}$ and $D_{\mathrm{s}}$ differ by approximately four orders of magnitude.

The domain is assumed to be isothermal, which can be justified by the large ratio of the thermal to the mass diffusivity. Since we are primarily interested in ripening (as opposed to solidification), the system is taken to be adiabatic, such that the following energy balance holds:

$$
\rho c_{p} \dot{T}=L \dot{f}_{\mathrm{s}}=L\langle\dot{\phi}\rangle
$$

where the dot denotes a derivative with respect to time, $\rho c_{p}$ is the solid/liquid mixture heat capacitance, $L$ is the latent heat, $f_{\mathrm{s}}=1-f_{1}$ is the solid volume fraction in the domain, and $\langle\dot{\phi}\rangle$ is the average rate of change of the phase-field variable over the entire domain. Note that the temperature in the system adjusts only due to changes in the mean curvature of the particles, according to the GibbsThomson condition. These adjustments are very small, resulting in an approximately constant solid fraction. Nonetheless, for consistency we did solve the above energy balance in conjunction with the transport equations of the previous section.

Since the primary solidification process is not simulated, the initial temperature, liquid velocities, and concentration distributions in the solid and liquid phases have to be specified. For this purpose, a certain initial solid fraction, $f_{\mathrm{s}}$, is first chosen for the domain. Then, the initial concentration in the liquid is assumed to be uniform and given by the Scheil equation, i.e.

$$
C_{1}=\frac{C_{0}}{\left(1-f_{\mathrm{s}}\right)^{1-k}} .
$$

The initial temperature is taken to be the equilibrium liquidus temperature corresponding to the initial liquid concentration from the Scheil equation.
The initial radial concentration profiles in the spherical solid particles were also calculated from the Scheil equation by varying the solid fraction from zero to the initial solid fraction. This results in an initial spatially nonuniform concentration distribution in the solid particles that is independent of the solidification path. Since the flow develops quickly relative to the total ripening time simulated, the initial velocity was simply set to zero.

The boundary conditions are as follows. The flow is driven by a constant pressure drop $\Delta p$ imposed at the north-south boundaries, such that the flow is from the top to the bottom of the domain. The concentration and phase-field variables are assumed to have a zero gradient at the east and west boundaries. Periodic boundary conditions are applied for all dependent variables at the north and south boundaries.

Numerical solutions to the transport equations were obtained using a standard control volume discretization scheme, with explicit time stepping for the phase-field equation and an implicit scheme for $C, \mathbf{v}_{1}$, and $p_{1}$. The momentum equation was discretized on a staggered grid and the velocity-pressure coupling was achieved using the Simple algorithm [40]. The simulations were performed on a $301 \times$ 301 uniform grid and for a sufficiently long time to achieve self-similar ripening behavior and until the number of particles became too small to allow for an analysis of the mean ripening behavior. The interface thickness parameter $\delta$ was chosen to be of the order of the grid size, but small compared to the average radii of the particles (see Table 1). In this case, and for motion mainly driven by the mean curvature, the solution of the phase-field equation was found to be independent of the interface thickness. For numerical reasons the interface mobility $\mu$ was chosen artificially small (see Table 1). In view of the extremely small interface velocities present during ripening, it can be understood that this choice had no effect on the computed results.

A stringent test problem for the present model (without phase change) is given by Stokes flow through regular arrays of infinite cylinders as shown in Fig. 1. Unit cells containing both square and triangular arrangements are considered. Analytical expressions for the drag force on the cylinders as a function of the solid fraction, $f_{\mathrm{s}}$, in the unit cell have been obtained by Sangani and Acrivos [11] and Drummond and Tahir [12]. The distribution of the phase-field variable $\phi$ was set before a computational run using a radially symmetric tangent hyperbolic profile to affect the smearing of the (stationary) solid/liquid interface. This is illustrated by the variation of gray tones in Fig. 1. A square grid of $51 \times 51$ control volumes was utilized in the simulations for the square array, and $52 \times 45$ for the triangular array. The interface thickness corresponded to about five control 


\section{square array}

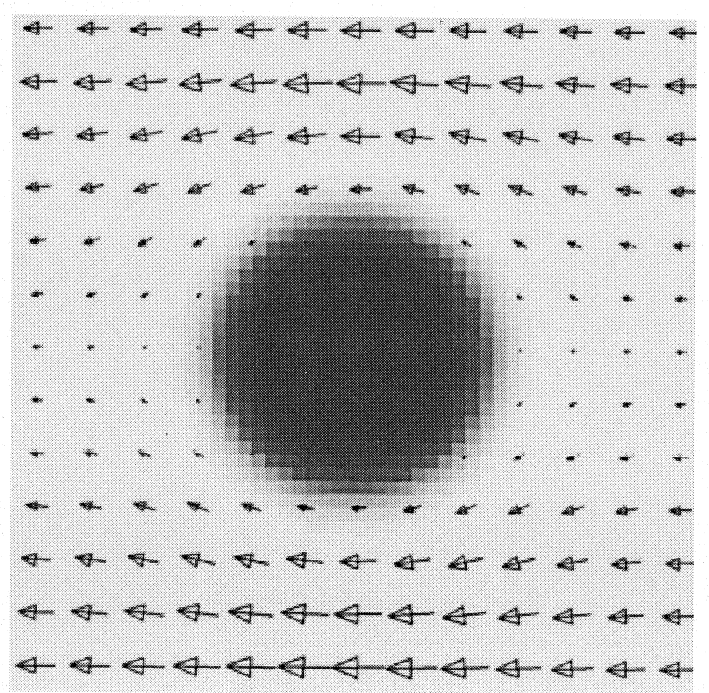

triangular array

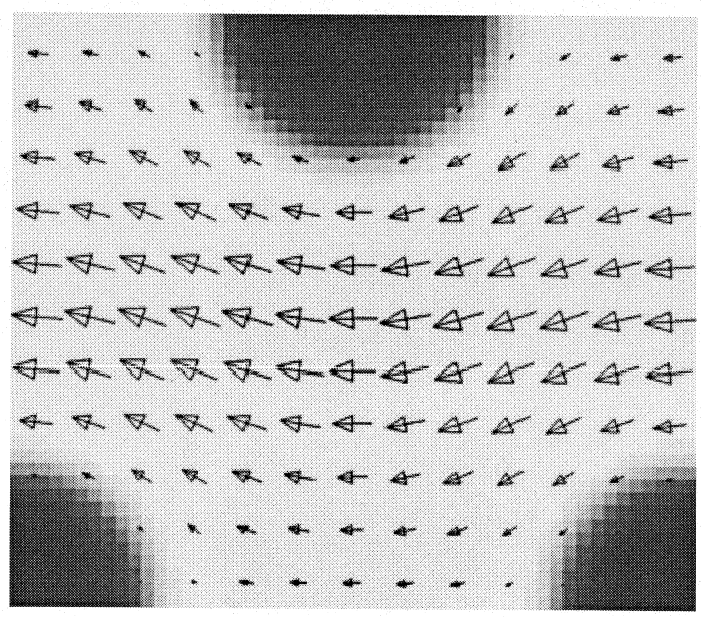

Fig. 1. Unit cells and sample computed velocities for the simulation of flow through regular arrays of infinite cylinders; the flow is periodic in the east-west direction and the phase-field distribution is indicated by the gray levels: (a) square array; (b) triangular array.

volumes. The numerical results, together with the analytical predictions, are plotted in Fig. 2. The mean liquid velocity through the unit cell is normalized by the pressure drop per unit width (drag force) and $4 \pi \eta$. It can be seen that the numerical results are generally in excellent agreement with the exact Stokes flow solutions. Minor deviations are present for small solid fractions, which can be attributed to the relatively coarse numerical grid used. For the smallest volume fraction, the diameter of the cylinders (measured at $\phi=0.5$ ) contains only six grid cells, implying that the diffuse interface thickness is of the same magnitude as the cylinder diameter. Nonetheless, remarkably accurate results are obtained. For the square array [Fig. 2(a)], large differences in the predictions occur for solid fractions greater than about 0.5 . This can be attributed to the fact that the analytical solutions break down in this regime. The present numerical method correctly predicts the mean velocity approaching zero when the packing fraction is reached. No such disagreement exists for the triangular array [Fig. 2(b)]. The computational results at high solid fractions, when the diffuse interfaces from neighboring cylinders almost overlap, show that the present model has excellent convergence properties for large interface thickness to flow passage width ratios.

Table 2 summarizes the conditions for the simulations of ripening, the results of which are presented and discussed in the following. Cases A, B, $\mathrm{C}$, and D correspond to (initial) solid volume fractions of $5.9,11.6,20.7$, and $29.2 \%$, respectively. Simulations were performed for each of these cases with and without convection. For the convection cases, the applied pressure drops $\Delta p$ are also listed in Table 2. Since the permeability decreases with increasing solid fraction, the pressure drop was increased from Case A to Case D in order to obtain mean flow velocities of a similar magnitude. For Case $\mathrm{B}$, three convection simulations with different pressure drops (B1, B2, and B3) are reported in order to investigate the dependence of the ripening kinetics on the flow velocity for the same solid fraction.

\section{RIPENING IN A DIFFUSIVE ENVIRONMENT}

Figure 3 shows the evolution of the phase field for Cases A-D without convection, starting from the initial distribution up to $t=5000 \mathrm{~s}$. It can be seen that for all cases the number of particles decreases strongly, while the particle size increases (i.e. the solid fraction is approximately constant). For the two higher solid fractions, coalescence processes can be occasionally observed. In that case, the shape of the particles becomes highly nonspherical. Otherwise, especially at the lower solid fractions, the particles retain an almost spherical shape at all times.

An example of the evolution of the computed solute concentration field is shown in Fig. 4 for Case C. In order to better visualize the concentration gradients, different gray scales are used for the solid and the liquid phases. The solid/liquid interface is indicated as a solid line. The results without convection are shown in the upper panels [Figs 4(a)-(d)]. The concentration gradients in the liquid are considerably lower than in the solid because of the large disparity in the diffusion coefficients. In fact, the diffusion in the solid is so slow that the concentration distribution in the solid can be regarded as a history of the interfacial curvature evolution according to the Gibbs-Thomson condition. The concentration gradients in the liquid are 


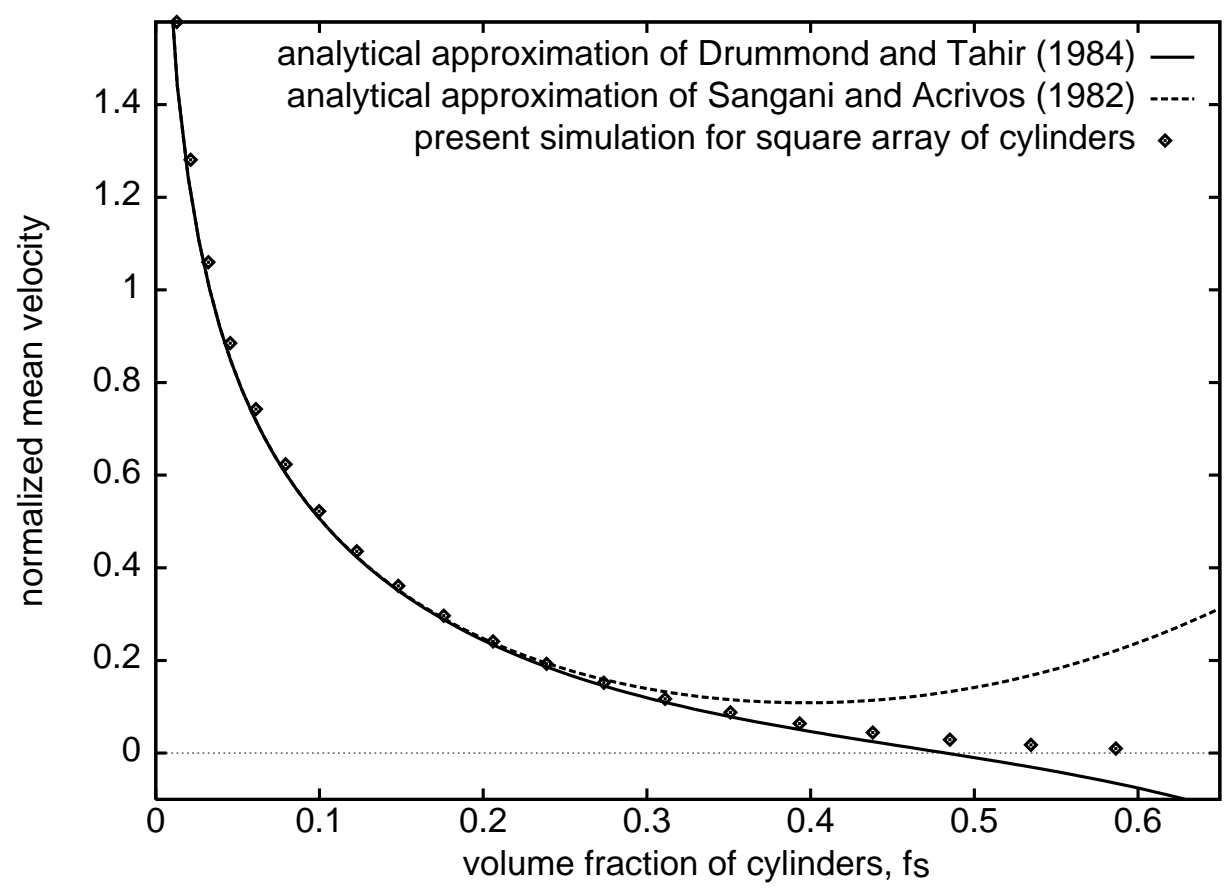

(a) square array

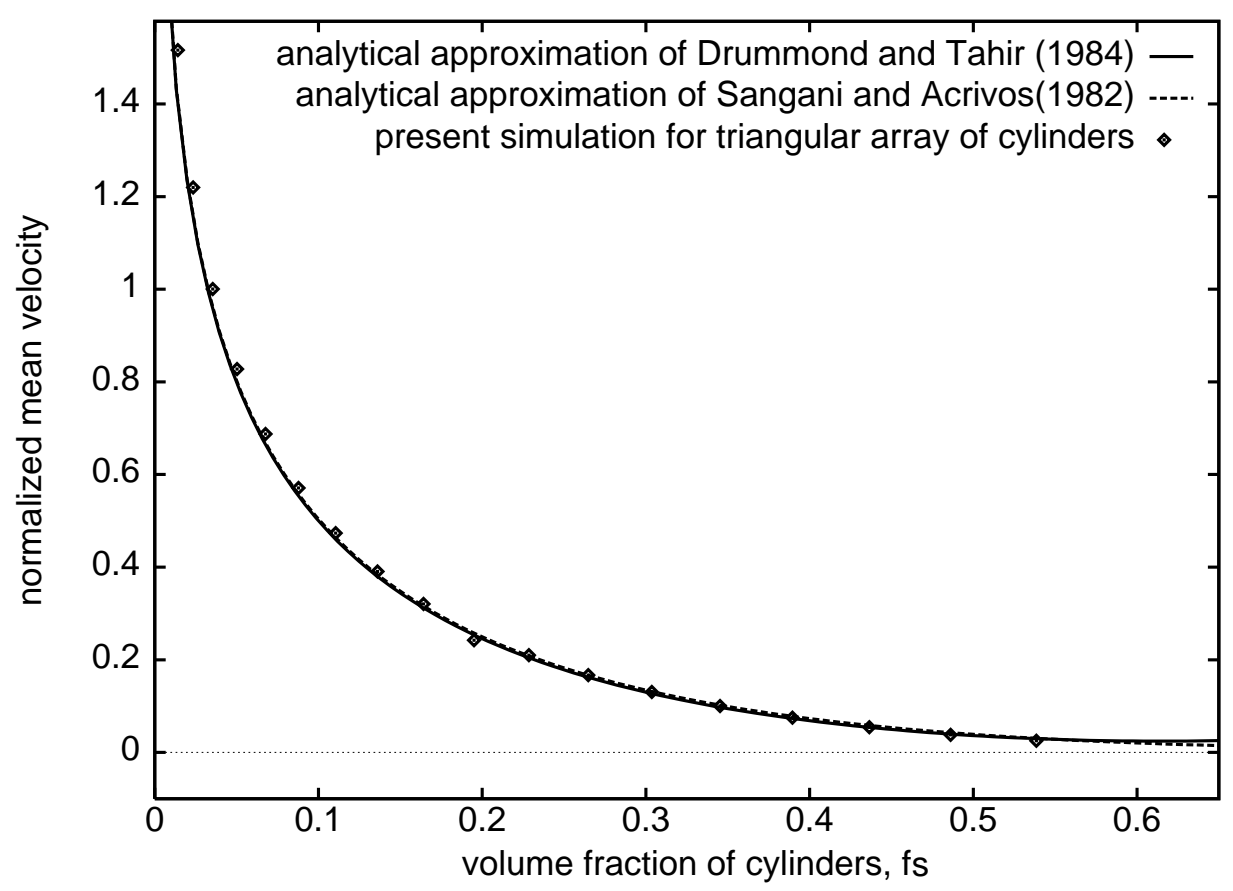

(b) triangular array

Fig. 2. Comparison of the computed mean velocities through the regular arrays of cylinders of Fig. 1 as a function of the volume fraction of cylinders with the analytical results of Sangani and Acrivos [11] and Drummond and Tahir [12]: (a) square array; (b) triangular array. 
generally the highest around the smallest particles, since they have the largest curvature and therefore the highest melting rate. High concentrations are present in the regions where two particles coalesce. This is caused, according to the Gibbs-Thomson condition, by the negative curvatures corresponding to the concave shape of the solid/liquid interface in the coalescence region. At later times, regions of high solute concentration due to coalescence can be observed inside a number of the larger particles.

Figure 5(a) shows the evolution of the mean radius for Case $\mathrm{C}\left(f_{\mathrm{s}}=20.7 \%\right)$. The corresponding plots for Cases $\mathrm{A}, \mathrm{B}$, and $\mathrm{D}$ are similar and not included here. The procedure used to evaluate the mean radius from the computed phase-field distributions is explained in Ref. [41]. It can be seen that after some time, the slope of the mean radius evolution for diffusion-controlled ripening approaches a value of $1 / 3$ on the $\log -\log$ plot. This is in agreement with the classical ripening exponent of $1 / 3$ in equations (2) and (3). The fluctuations in the mean radius evolution apparent in Fig. 5(a) are due to particles disappearing and coalescing, and cannot be avoided with the limited number of particles employed in the present simulations. The fluctuations become stronger with time, because the particle number decreases continually (see Fig. 3 ). The same problem exists when plotting the normalized radius distribution, as shown in Fig. 5(b) for Case $\mathrm{C}$ at $2000 \mathrm{~s}$. The method used for evaluating the radius distribution is also explained in Ref. [41]. For easy reference, the initial distribution and the distribution according to LSW theory (as well as the flow results; see below) are included in Fig. 5(b). It can be seen that the distribution for the purely diffusive case remains fairly close to the initial distribution, which was taken to be the asymptotic distribution from the two-dimensional ripening theory (without flow) of Marqusee [20]. The maximum of the computed distribution appears to shift toward smaller radii and a relatively long tail is established beyond 1.6. The tail is due to coalescence of particles, because the radii can be very large (and even negative) for the solid neck connecting two coalescing particles. The computed radius distributions for the lower solid fraction cases $\mathrm{A}$ and B (not shown here) do not exhibit such a tail because no coalescence occurs (see Fig. 3).

Aside from the mean radius, the specific surface area, $S_{\mathrm{V}}$, is another quantity that allows for an examination of the ripening kinetics. It is obtained from the computed phase-field distribution by integrating $|\nabla \phi|$ over the domain [41]. The results for all four cases are shown in Fig. 6(a), with the asymptotic behavior for Case $\mathrm{C}$ magnified in Fig. 6(b). Because the specific surface area is an integral quantity for the simulation domain, its time evolution is much smoother, even for small numbers of particles, than the one for the mean radius (cf. Figs 5 and 6). As can be seen from Fig. 6, the specific surface area thus allows for a less ambiguous evaluation of the mean ripening kinetics. For the diffusion simulations, the long-time asymptotic behavior of the specific surface area shows the expected ripening exponent of $-1 / 3$ for all four solid fractions. The comparison with the flow results is discussed in the next section.

Finally, we have used the present results for the evolution of the mean radius to estimate the ripening rate constant $K_{\text {diff }}$ in equations (2) and (3) for the four diffusion simulations, by assuming a ripening exponent of $1 / 3$. For easy comparison with other theories, the rate constants are then nondimensionalized using the following equation from Voorhees [18]:

$$
K_{\text {diff }}=K_{\text {diff }}^{*} \frac{(\operatorname{dim}-1) \Gamma D_{1}}{(1-k) m_{1} C_{0}}
$$

where $K_{\text {diff }}^{*}$ is the dimensionless diffusion rate constant and dim is the dimensionality of the system ( $\operatorname{dim}=2$ here). Note that according to LSW theory, $K_{\text {diff }}^{*}=4 / 9$ for diffusive ripening of spheres in three dimensions and in the limit of vanishing solid fraction. The dimensionless rate constants, $K_{\text {diff }}^{*}$, estimated from the present simulations are listed in Table 2 and plotted as a function of the solid volume fraction in Fig. 7. It can be seen that the present rate constants are generally in good agreement with the monopole (M) and dipole (D) approximate numerical results of Akaiwa and Meiron [26] and the two-dimensional theory of Marqusee [20]. In accordance with the previous results [20, 26], the rate constant increases with increasing solid fraction. Any differences with the previous results are well within the uncertainties introduced by the limited number of particles used in the present simulations, or could be caused by coalescence of particles in the higher solid fraction cases.

Table 2. Summary of simulation cases and results for the scaled ripening rate constants and permeabilities

\begin{tabular}{lcccccc}
\hline Case & $f_{\mathrm{s}}(\%)$ & $K_{\text {diff }}^{*}$ & $\Delta p\left(\mathrm{~N} / \mathrm{m}^{2}\right)$ & $K_{\text {conv }}^{*}$ & $\Pi S_{\mathrm{V}}^{2}$ & $\Pi R^{2}$ \\
\hline A & 5.9 & 0.32 & 0.1 & 1.34 & 11.7 & 3.6 \\
B1 & 11.6 & 0.41 & 0.2 & 1.79 & 3.6 & 1.09 \\
B2 & 11.6 & 0.41 & 0.5 & 1.60 & 3.6 & 1.09 \\
B3 & 11.6 & 0.41 & 0.2 & 2.52 & 0.85 & 0.09 \\
C & 20.7 & 0.60 & 0.3 & 3.80 & 0.32 & 0.09 \\
D & 29.2 & 1.08 & & & & 0.09 \\
\hline
\end{tabular}




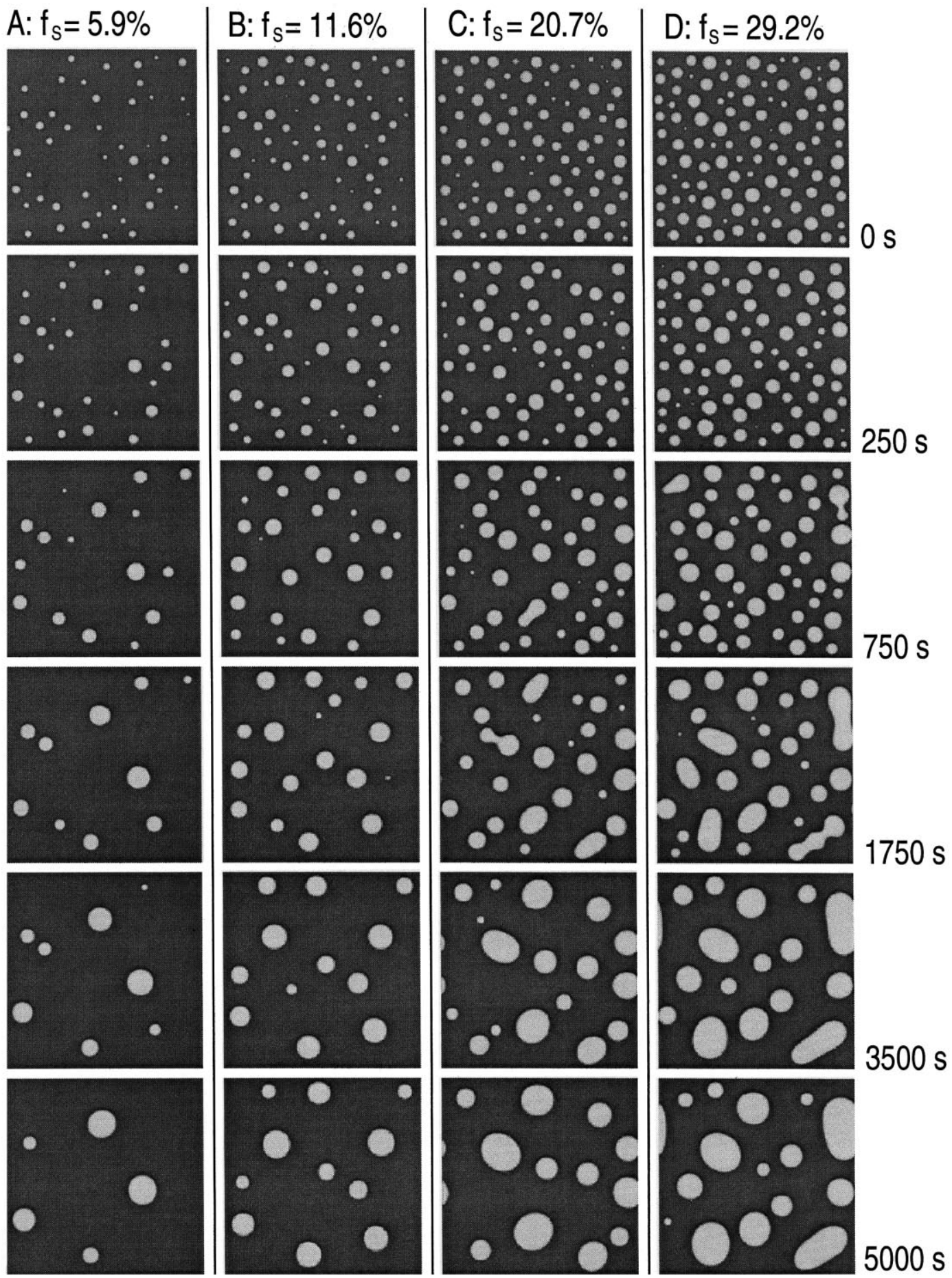

Fig. 3. Predicted phase-field evolution for the four ripening simulations without convection; the value of the phase-field variable is indicated by a continuous gray scale, where $\phi=0$ (liquid) is black and $\phi=1$ (solid) is white.

\section{RIPENING IN A CONVECTIVE ENVIRONMENT}

An example of computed phase fields, solute concentrations, and liquid velocities for ripening with convection is shown in Figs 4(e)-(h) (lower panels) for Case $\mathrm{C}$ at the same times as the corresponding pure diffusion results [Figs 4(a)-(d); upper panels].
Considerable differences between the no-flow/flow cases are apparent in the evolution of the particle shapes and sizes. These differences are due to advection of solute by the flow. As the particles coarsen, the liquid velocities increase strongly. This can be attributed to the drag being reduced at later times 
due to the decreased specific surface area or increased hydraulic radius, while the applied pressure drop is held constant.

Figure 5(a) indicates that in the presence of convection the mean radius of the particles increases progressively faster than under purely diffusive conditions. At later times, the liquid velocities become high enough to accelerate the ripening process, such that the ripening exponent for the convection simulation tends toward a value of $1 / 2$, as schematically indicated in Fig. 5(a). The ripening theory developed by Ratke and Thieringer [22] shows that in the presence of convection the mean radius increases according to

$$
R^{2}-R_{0}^{2}=K_{\text {conv }} t
$$

where $K_{\mathrm{conv}}$ is the convection ripening rate constant. In the long-time limit, we thus have $R-t^{1 / 2}$. The theory of Ratke and Thieringer [22] was originally intended for ripening of freely falling spheres under the assumption of Stokes flow $\left(U-R^{2}\right)$. Although the particles do not move in the system considered here, the analysis of Ratke and Thieringer can be used because the flow of the liquid through the simulated mush is also Stokes flow with respect to the mean particle radius. It is shown in the next section that Darcy's law, equation (1), indeed applies in the present convection simulations and that the permeablity scales with $\Pi-R^{2}$. The computed radius distribution at $2000 \mathrm{~s}$ for Case $\mathrm{C}$ with convection is shown in Fig. 5(b) and compared to the corresponding diffusion case. The analytically determined distribution of
Ratke and Thieringer [22], valid for three dimensions and in the limit of zero solid fraction, is included for reference. As already discussed in the previous section, the number of particles is too small to allow for a quantitative analysis of the radius distribution obtained from the present simulation. Qualitatively, it may be seen that the distribution from the convection simulation is somewhat broader than in the diffusion case. This trend is supported by the theory of Ref. [22].

The change in the ripening exponent due to convection is more obvious in the plots of the evolution of the specific surface area of Fig. 6. Results for all four cases (A-D) are included in Fig. 6(a). In particular, the magnified plot of Fig. 6(b) for Case C clearly shows that convection causes the ripening exponent for the specific surface area to change from $-1 / 3$ to $-1 / 2$ in the long-time limit.

The ripening rate constants in the convection cases, $K_{\text {conv }}$, can be estimated by plotting $R^{2}-R_{0}^{2}$ against $t$, which is demonstrated in Fig. 8 for Cases B1, B2, and B3. Despite the strong fluctuations due to the limited number of particles, it can be seen that the mean radius evolution indeed approaches a $R^{2}-t$ behavior. Figure 8 also allows for an examination of the effect of the applied pressure drop and, hence, the mean flow velocity on the ripening rate at constant solid fraction (Cases B1, B2, and B3 all have solid fractions of $11.6 \%$ ). It can be seen that the convection rate constant increases with the applied pressure drop.

The ripening rate constants for the convection cases can be analyzed in more detail by scaling
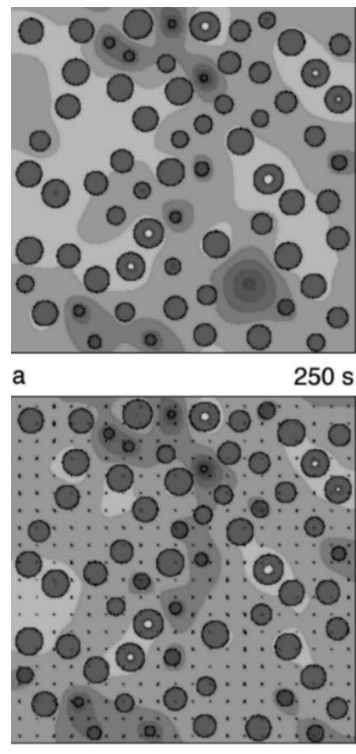

e

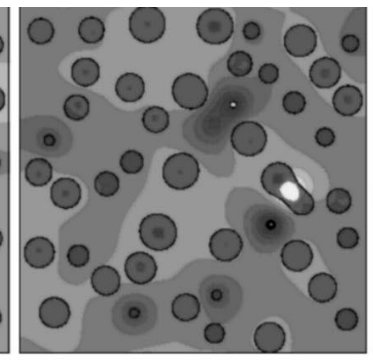

750 s c

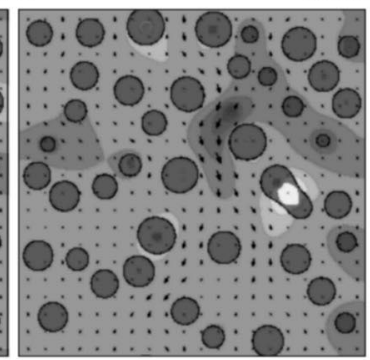

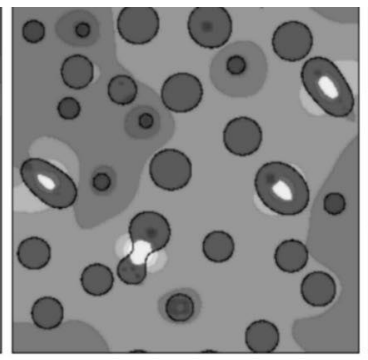

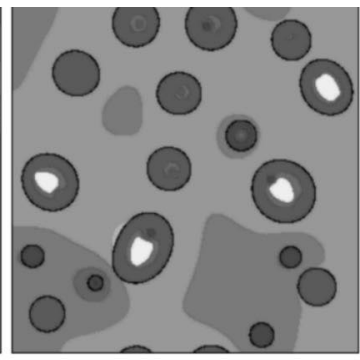

1750 s d

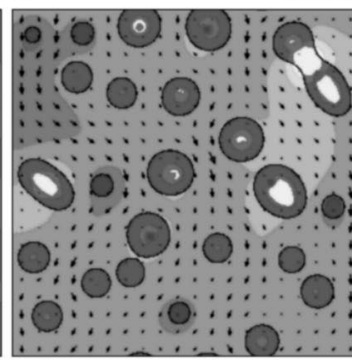

g

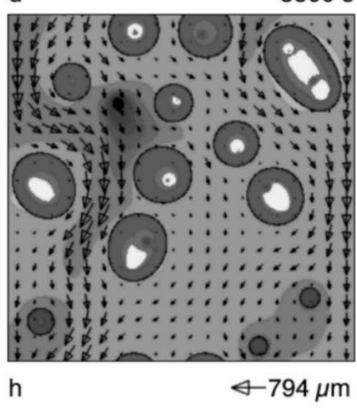

Fig. 4. Evolution of the predicted solute concentration fields (gray scale), liquid velocities (arrows), and phase field (a black contour line is drawn at $\phi=0.5$ ) for Case $\mathrm{C}$; for the solute concentration in the liquid, a gray scale with ten equidistant intervals between 4.861 and $4.871 \mathrm{wt} \% \mathrm{Cu}$ is used, while four intervals between 0.682 and $0.6805 \mathrm{wt} \% \mathrm{Cu}$ are used for the solid: (a)-(d) (upper panels) diffusion-controlled ripening; (e)-(h) (lower panels) ripening with convection. 
(a)

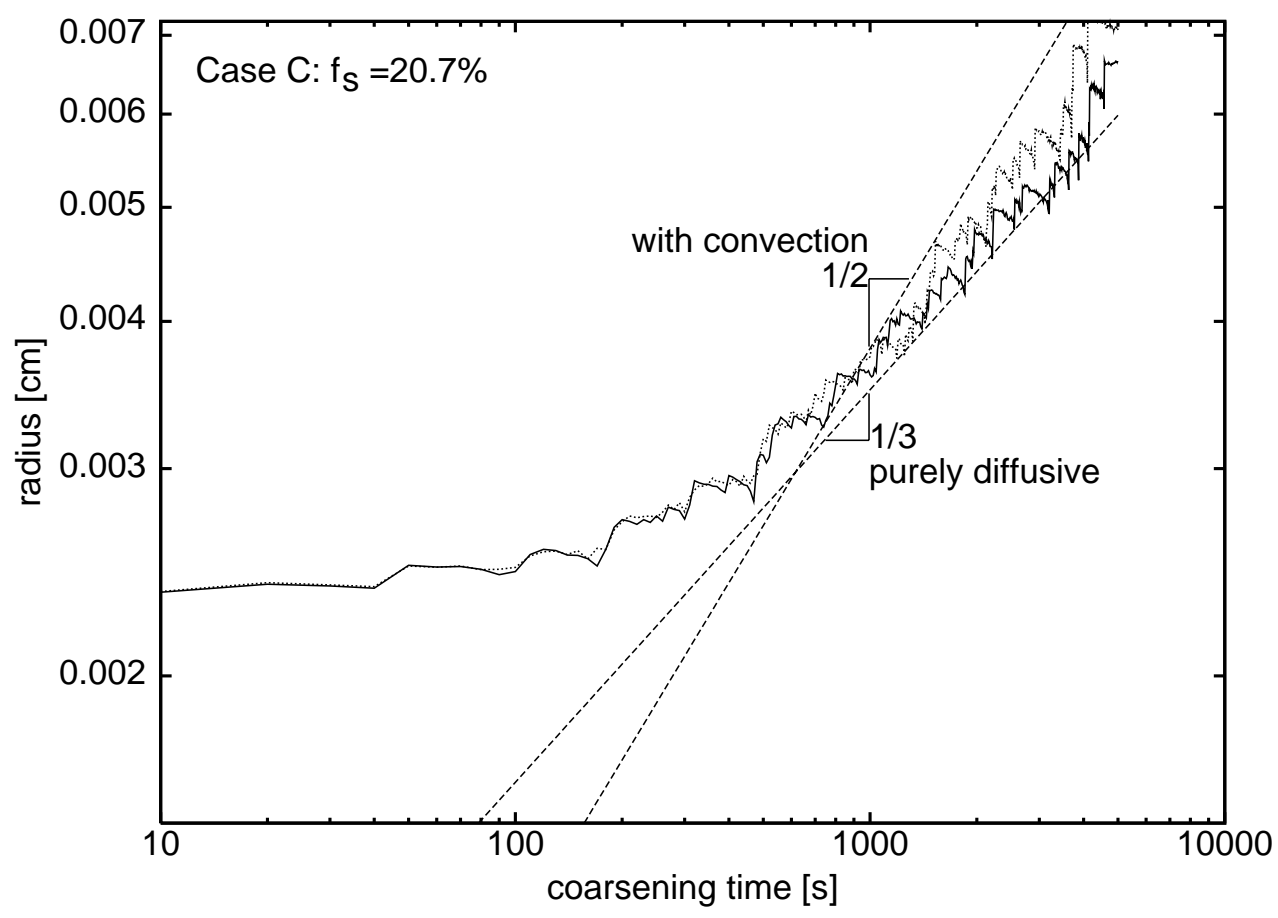

(b)

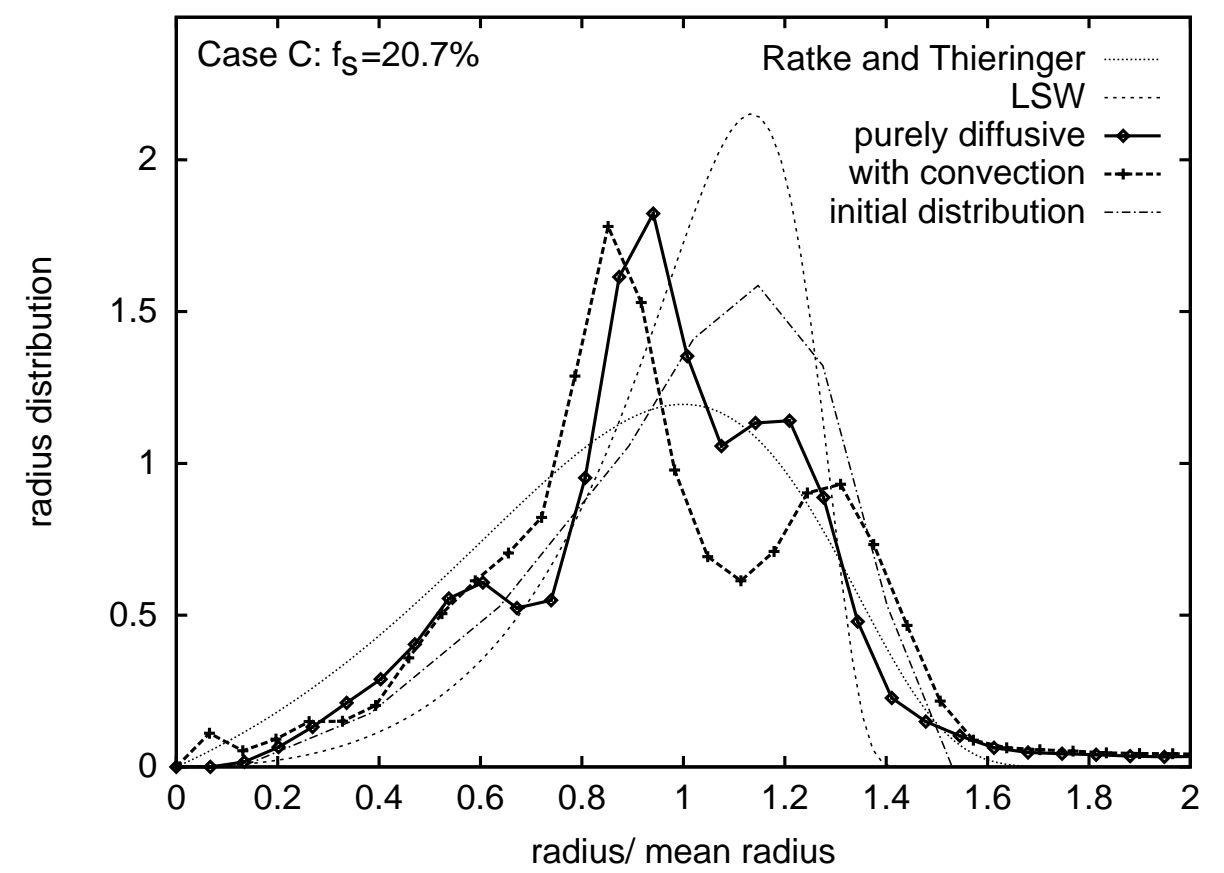

Fig. 5. Results for the predicted particle radii in Case $\mathrm{C}$ with and without convection: (a) evolution of the mean radius; (b) normalized radius distribution (simulation results are at $2000 \mathrm{~s}$ ).

them using the theory of Ratke and Thieringer [22]. According to that theory, a dimensionless convection rate constant, $K_{\text {conv }}^{*}$, can be defined as

$$
K_{\text {conv }}=\frac{K_{\text {conv }}^{*}}{2 \pi} \frac{(\operatorname{dim}-1) \Gamma D_{1}}{(1-k) m_{1} C_{0}}\left[\frac{U}{D_{1} R^{2}}\right]^{1 / 3}
$$

where $U$ is the relative velocity between the solid and liquid. Note that $U-R^{2}$ for Stokes flow, such that the rate constant is in fact independent of the mean radius. Ratke and Thieringer determined that $K_{\text {conv }}^{*}=1.575$ for convective ripening of spheres in three dimensions and in the limit of a 
vanishing solid fraction. For the flow through the mush of the present system, the relative velocity $U$ is given by Darcy's law which, by integration of equation (1) over the length $L$ of the system, can be written as

$$
U=\Pi \frac{\Delta p}{L \eta} .
$$

Hence, the main result of this theory is that the dimensional rate constant $K_{\text {conv }}$ is proportional to $\left[\left(\Pi / R^{2}\right) \Delta p\right]^{1 / 3}$. It is shown in the next section that
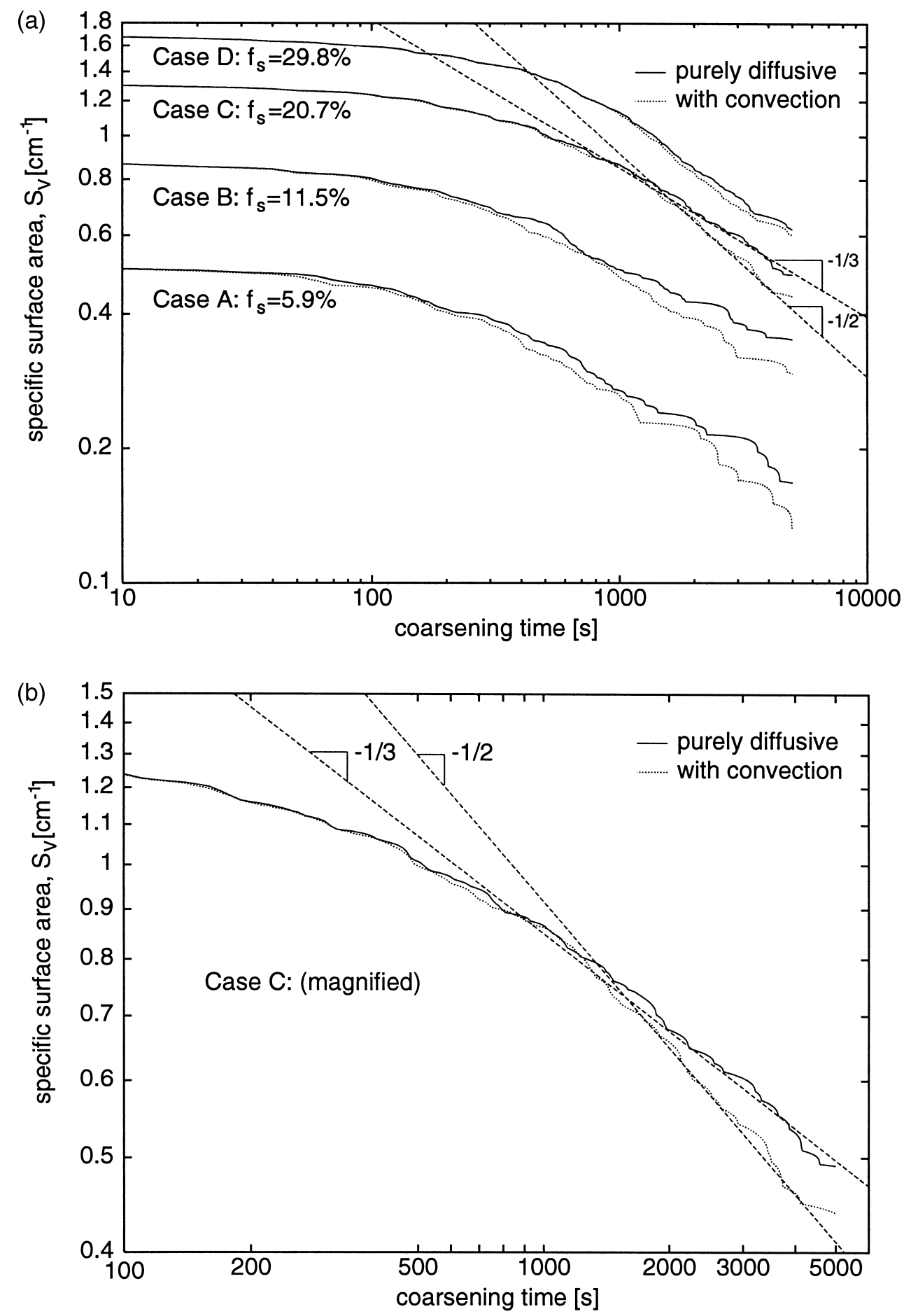

Fig. 6. $\log -\log$ plots of the predicted specific surface area evolution for Cases A-D with and without convection: (a) comparison of all cases; (b) magnified plot for Case C. 
the normalized permeability, $\Pi / R^{2}$, is independent of the applied pressure drop, as one would expect from Darcy's law, and constant in time. Therefore, for an increase in the pressure drop by a factor of five, going from Case B1 to Case B3, the dimensional rate constant should increase by a factor of $5^{1 / 3}=1.71$. An examination of the slopes of the straight lines sketched in Fig. 8 shows an increase in the dimensional rate constant by a factor of approximately 1.5 going from Case B1 to Case B3, which is in reasonable agreement with the theory considering the uncertainties introduced by the limited number of particles.

Scaling the ripening rate constants for all convection simulations using equations (12) and (13), results in the dimensionless rate constants $K_{\text {conv }}^{*}$ listed in Table 2. These values are plotted as a function of the solid volume fraction in Fig. 9. The dimensionless rate constants increase with increasing solid fraction in a manner similar to the diffusion cases (see Fig. 7). Furthermore, it can be seen that the dimensionless rate constants for Cases B1, B2, and B3, which differ only in the applied pressure drop, are indeed close to each other. A theory that predicts the observed increase of the convection rate constants with solid fraction is, to the authors' best knowledge, not available in the literature.

\section{EVALUATION OF THE PERMEABILITY}

As discussed in Section 1, the direct numerical simulation of the flow through the mush allows for the evaluation of the permeability, $\Pi$, as defined by Darcy's law, equation (1). Here, it is of particular interest to examine the effect of ripening on the permeability. The permeability was determined by measuring the mean velocity, $U$, for the computational domain from the predicted liquid velocities and substituting it together with the applied pressure drop, $\Delta p$, into equation (13).

Figure 10(a) shows the predicted evolution of the permeability for all simulation cases. The permeability increases with time because ripening reduces the drag by decreasing the specific surface area or increasing the hydraulic radius. This effect is particularly significant for the lower solid fraction cases (A and B). The rate of increase of the permeability decreases with time because the ripening rate decreases (i.e. $\mathrm{d} R / \mathrm{d} t-t^{-1 / 2}$ ). It is important to remember that the solid volume fraction is approximately constant in time for each case, implying that the variations in the permeability are due to ripening only. The fluctuations in the curves are again due to the disappearance or coalescence of particles.

An important issue in determining the permeability of the mush in solidification systems is the choice of a suitable microstructural length scale,

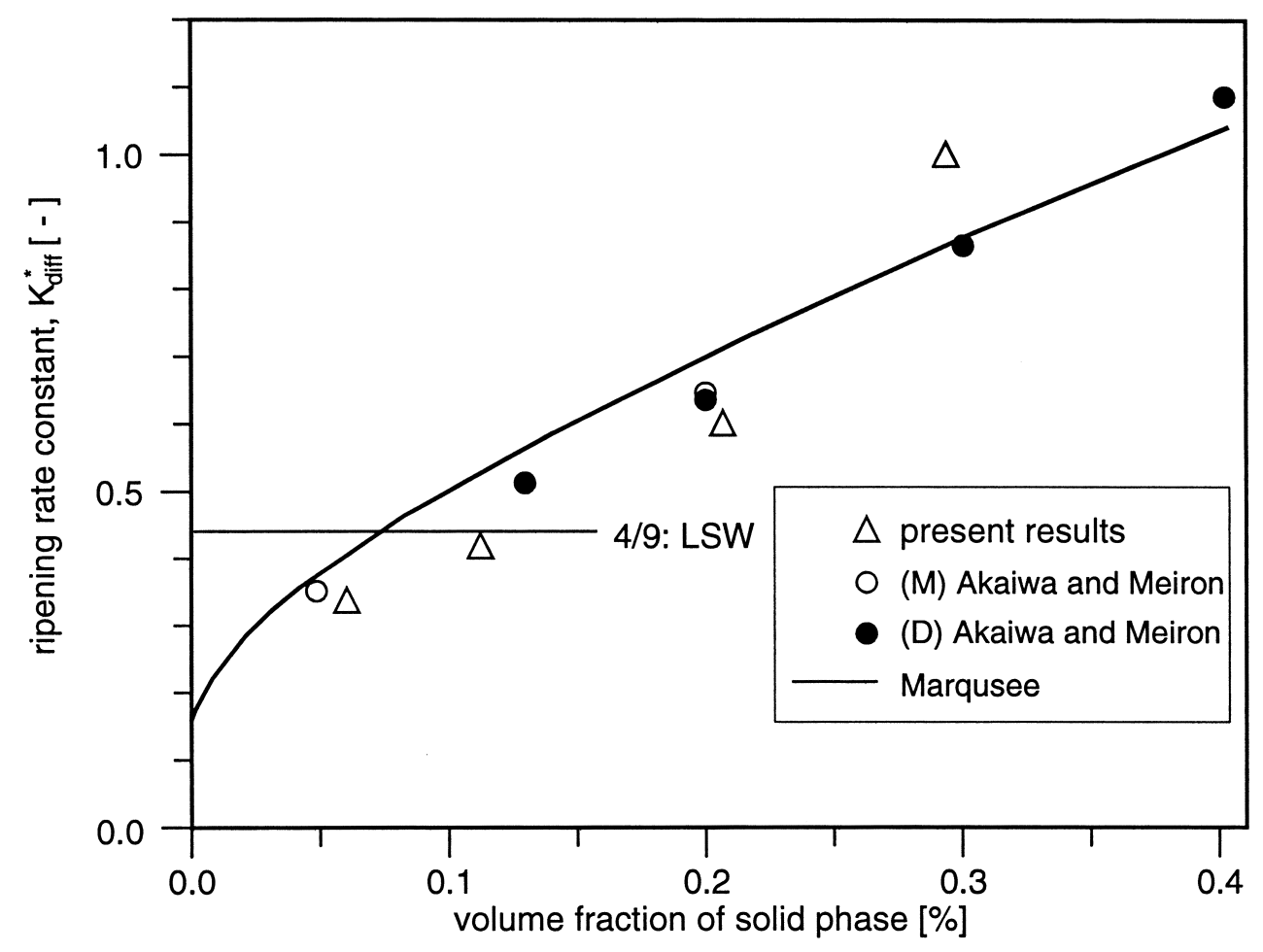

Fig. 7. Predicted dimensionless rate constants as a function of the solid volume fraction for the diffusion-controlled ripening cases, and comparison with the theory of Marqusee [20] and the numerical results of Akaiwa and Meiron [26]. 


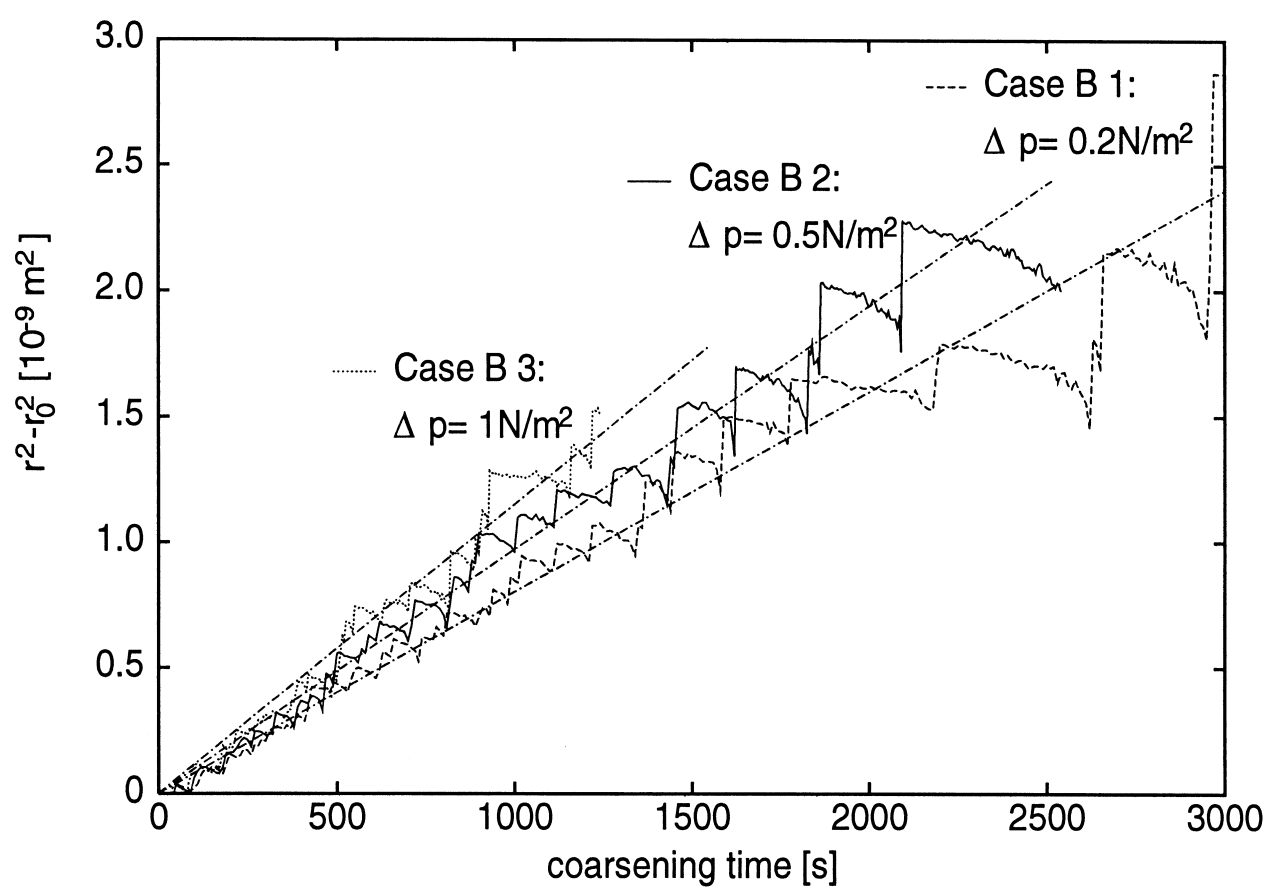

Fig. 8. Predicted evolution of the squared mean radius for the ripening simulations with convection of Cases B1, B2, and B3; these cases differ only in the applied pressure drop.

such that the scaled permeability is the same at a given solid volume fraction regardless of the geometry of the microstructure. The obvious choices for such a length scale in the present system are the mean radius, $R$, and the specific surface area, $S_{\mathrm{V}}$, both of which evolve with time. Figure 10(b) shows the variation of the scaled permeabilities $\Pi / R^{2}$ (lower curve) and $\Pi S_{\mathrm{V}}^{2}$ (upper curve) for Case D. After some initial transient, both of the scaled permeabilities remain constant other than for the una-

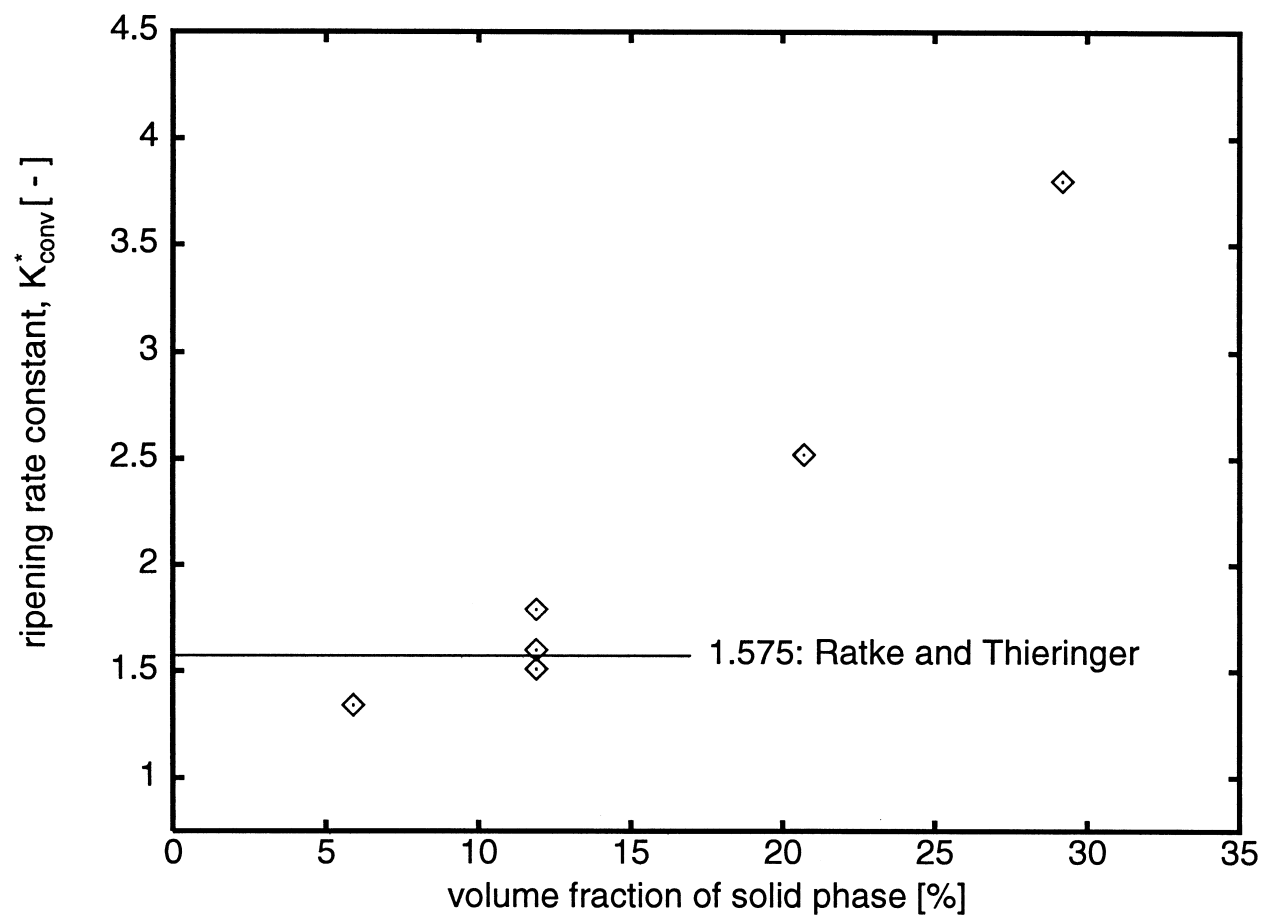

Fig. 9. Predicted dimensionless rate constants as a function of solid volume fraction for all ripening simulation cases with convection. 

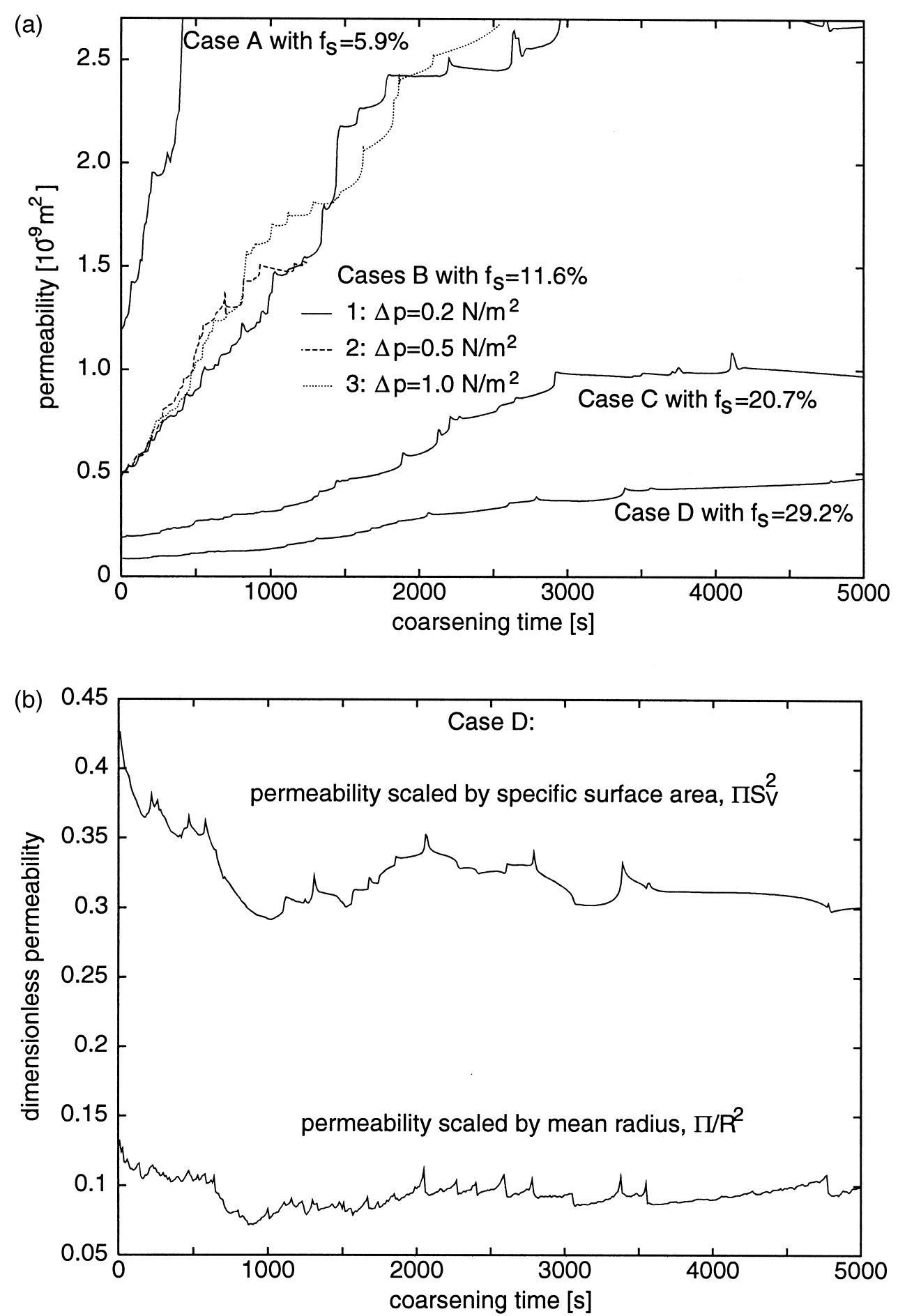

Fig. 10. Evolution of the measured permeabilities for all ripening simulation cases with convection: (a) dimensional permeability; (b) scaled permeabilty.

voidable fluctuations due to the limited number of particles. This shows not only that Darcy's law is applicable in the present simulations, but also that either scaling succeeds in predicting the effect of ripening on the permeability.
Taking an average over time, a single value of the scaled permeabilities can be determined for each of the convection cases. All of these values are listed in Table 2, and the values for $\Pi S_{\mathrm{V}}^{2}$ are additionally plotted in Fig. 11 as a function of the 


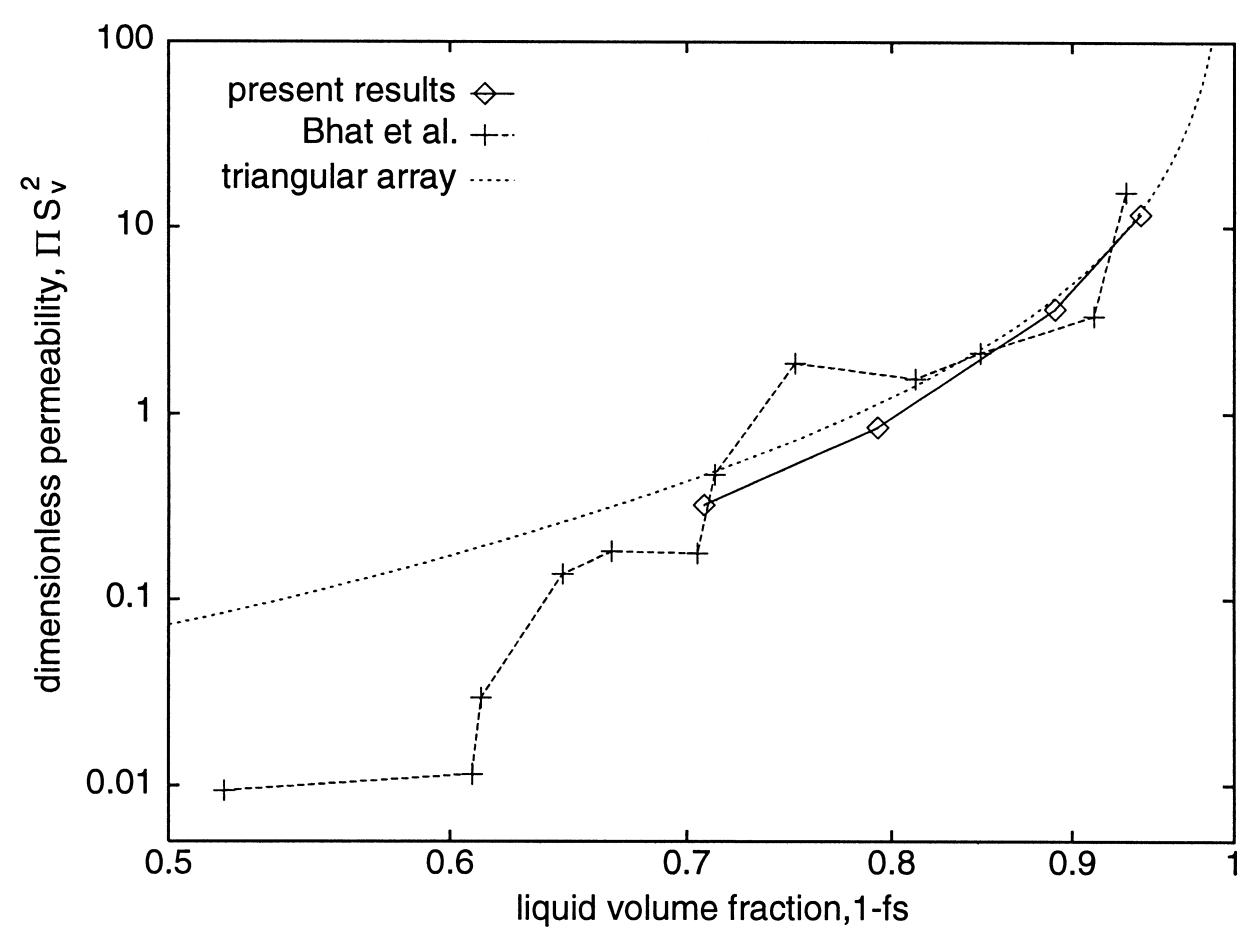

Fig. 11. Predicted permeabilities scaled with the specific surface area as a function of the liquid fraction for all ripening simulation cases with convection, and comparison with the analytical results of Drummond and Tahir [12] for a triangular array of cylinders and the numerical results of Bhat et al. [8] for a measured columnar dendritic microstructure.

liquid volume fraction, $1-f_{\mathrm{s}}$. As expected, the scaled permeabilities for Cases B1, B2, and B3 are identical to within the present measurement uncertainties and only one mean value is listed. Also included in Fig. 11 are the analytical results of Drummond and Tahir [12] for Stokes flow through a triangular array of equal-sized cylinders and the numerical results of Bhat et al. [8] for two-dimensional flow through a cross section of a measured columnar dendritic microstructure. It can be seen that for large liquid fractions $(>0.8)$, all results agree with each other. This indicates that the scaled permeabilities are independent of the ripening kinetics, because the previous results are all for nonevolving, fixed microstructures. For decreasing liquid fraction, both the present scaled permeabilities and the results of Bhat et al. [8] are increasingly lower than the permeabilities for the triangular array of cylinders. More detailed comparisons are not possible in this regime due to the large scatter of the data of Bhat et al.

\section{CONCLUSIONS}

The interactions between ripening and melt flow in a solidification microstructure or mush of an $\mathrm{Al}-$ $\mathrm{Cu}$ alloy have been investigated numerically using the phase-field method. The accuracy of the present phase-field method with convection is demonstrated by comparing predictions of Stokes flow through arrays of cylinders with analytical solutions. A detailed parametric study is performed to investigate the ripening kinetics both for diffusion and convection cases with various volume fractions of solid in the domain. The results for the diffusion cases, both in terms of the ripening exponent and the dependence of the ripening rate constant on the solid fraction, are shown to be in good agreement with previous theories of ripening. At higher solid fractions, coalescence of particles is observed. The limited number of particles in the present computations hinders a quantitative analysis of the particle size distributions at long ripening times.

The simulation cases with convection show that the ripening exponent changes from the classical value of $1 / 3$ for diffusion controlled ripening to $1 / 2$ as the flow velocities increase due to the coarsening of the structure. This, as well as the predicted trend toward a broader radius distribution, is in agreement with the convective ripening theory of Ratke and Thieringer [22]. The ripening rate constants estimated from the present convection simulations are scaled using the Ratke-Thieringer theory, resulting in dimensionless rate constants that depend on the solid volume fraction only. These results still have to be analyzed theoretically.

The simulation results are also used to investigate the effect of ripening on the permeability of the mush, as defined in Darcy's law. It is shown that ripening causes a continual increase of the permeability with time. This effect can be predicted by scaling the permeability with the mean radius or the 
specific surface area. The scaled permeabilities are in quantitative agreement with previous results for fixed (time invariant) microstructures.

An obvious extension of the present study is to simulate a non-adiabatic system, such that ripening and solidification occur simultaneously. The investigation of the effect of melt convection in such a system is of great practical interest. Extension of the simulations to three dimensions is also desirable, but must await the development of more efficient numerical techniques and/or faster computers.

Acknowledgements - This work was supported by the National Science Foundation (NSF) under grant No CTS-9501389 and NASA under contract NCC8-94. We thank L. Ratke of the DLR, Germany, for many helpful suggestions.

\section{REFERENCES}

1. Bennon, W. D. and Incropera, F. P., Int. J. Heat Mass Transfer, 1987, 30, 2161.

2. Beckermann, C. and Viskanta, R., Appl. Mech. Rev., 1993, 46, 1.

3. Voller, V. R., Brent, A. D. and Prakash, C., Int. J. Heat Mass Transfer, 1989, 32, 1719.

4. Nandapurkar, P., Poirier, D. R., Heinrich, J. C. and Felicelli, S., Metall. Trans., 1989, 20B, 883.

5. Bhat, M. S., Poirier, D. R. and Heinrich, J. C., Metall. Trans., 1995, 26B, 1091.

6. Ganesan, S., Chan, C. L. and Poirier, D. R., Mater Sci. Engng, 1992, 151A, 97.

7. Poirier, D. R., Metall. Trans., 1985, 18B, 245.

8. Bhat, M. S., Poirier, D. R. and Heinrich, J. C., Metall. Trans., 1995, 26B, 1049.

9. Bhat, M. S., Poirier, D. R., Heinrich, J. C. and Nagelhout, D., Scripta metall. mater., 1994, 31, 339.

10. Ostwald, W., Z. phys. Chem., 1900, 34, 495.

11. Sangani, A. S. and Acrivos, A., Int. J. Multiphase Flow, 1982, 8, 343.

12. Drummond, J. E. and Tahir, M. I., Int. J. Multiphase Flow, 1984, 10, 515.

13. McCarthy, J. F., Acta metall. mater., 1994, 42, 1573.
14. Lifshitz, I. M. and Slyozov, V. V., J. Phys. Chem. Solids, 1961, 19, 35.

15. Wagner, C., Z. Elektrochem., 1961, 65, 581.

16. Marsh, S. P. and Glicksman, M. E., Metall. Trans., 1996, 27A, 557.

17. Voorhees, P. W., J. Stat. Phys., 1985, 38, 231.

18. Voorhees, P. W., Metall. Trans., 1990, 21A, 27.

19. Ardell, A. J., Phys. Rev., 1990, 41B, 2554.

20. Marqusee, J. A., J. chem. Phys., 1984, 81, 976.

21. Yao, J. H., Elder, K. R., Guo, H. and Grant, M., Phys. Rev., 1993, 47B, 14110.

22. Ratke, L. and Thieringer, W. K., Acta metall., 1985 , 33, 1793.

23. Ratke, L., Mater. Sci. Engng, 1995, 203A, 399.

24. Akaiwa, N., Hardy, S. C. and Voorhees, P. W., Acta metall. mater., 1991, 39, 2931.

25. Wan, G. and Sahm, P. R., Acta metall. mater., 1990 , 38, 967.

26. Akaiwa, N. and Meiron, D. I., Phys. Rev., 1995, 51E, 5408.

27. Küpper, T. and Masbaum, N., Acta metall. mater., 1994, 42, 1847.

28. Langer, J. S., Directions in Condensed Matter. World Scientific, Singapore, 1986.

29. Kobayashi, R., Physica, 1993, 63D, 410.

30. Wang, S. L., et al., Physica, 1993, 69D, 189.

31. Karma, A. and Rappel, W. J., Phys. Rev., 1996, 53E.

32. Wheeler, A. A., Boettinger, W. J. and McFadden, G. B., Phys. Rev., 1992, 45A, 7424.

33. Warren, J. A. and Boettinger, W. J., Acta metall. mater., 1995, 43, 689.

34. Tiaden, J., Nestler, B., Diepers, H.-J. and Steinbach, I., Physica, 1998, 115D, 73.

35. Diepers, H.-J., Beckermann, C. and Steinbach, I., in Solidification Processing 1997, ed. J. Beech and H. Jones. Ranmoor House, Sheffield, 1997.

36. Beckermann, C., Diepers, H.-J., Steinbach, I., Karma, A. and Tong, X., J. Comp. Phys., in press.

37. Warren, J. A. and Murray, B. T., Model. Simul. Mater. Sci. Engng, 1996, 4, 215.

38. Kurz, W. and Fischer, D. J., Fundamentals of Solidification. Trans.Tech. Publications, Switzerland, 1992.

39. Rothwell, E., J. Inst. Metals, 1962, 90, 389.

40. Patankar, S., Numerical Heat Transfer and Fluid Flow. Hemisphere, Washington, DC, 1980

41. Diepers, H.-J., Diplom thesis, RWTH Aachen, Germany, 1998 